\title{
The effect of sidewall roughness on line edge roughness in top-down scanning electron microscopy images
}

\author{
T. Verduin, S. R. Lokhorst, P. Kruit, and C. W. Hagen \\ Delft University of Technology \\ Faculty of Applied Sciences \\ Department of Imaging Physics \\ Lorentzweg 1, 2628 CJ Delft, The Netherlands
}

\begin{abstract}
We have investigated in a numerical study the determination of sidewall roughness (SWR) from topdown scanning electron microscopy (SEM) images. In a typical metrology application, top-down SEM images are acquired in a (critical-dimension) SEM and the roughness is analyzed. However, the true size, shape and roughness characteristics of resist features are not fully investigated in the analysis of top-down SEM images. In reality, rough resist features are complex three-dimensional structures and the characterization naturally extends to the analysis of SWR.

In this study we randomly generate images of rough lines and spaces, where the lines are made of PMMA on a silicon substrate. The lines that we study have a length of $2 \mu \mathrm{m}$, a width of $32 \mathrm{~nm}$ and a height of $32 \mathrm{~nm}$. The SWR is modeled by using the power spectral density (PSD) function of Palasantzas, which characterizes roughness by the standard deviation $\sigma$, correlation length $\xi$ and roughness exponent $\alpha$. The actual roughness is generated by application of the method of Thorsos in two dimensions. The images are constructed by using a home-built program for simulating electron-specimen interactions. The program that we have developed is optimized for complex arbitrary geometries and large number of incident low energy primary electrons by using multicore CPUs and GPUs. The program uses the dielectric function model for inelastic scattering events and has an implementation specifically for low energy electrons. A satisfactory comparison is made between the secondary electron yields from the home-built program and another program found in literature. In order to reduce the risk of shrinkage, we use a beam energy of $300 \mathrm{eV}$ and a spot size of $3 \mathrm{~nm}$. Each pixel is exposed with 20 electrons on average $\left(\approx 276 \mu \mathrm{C} / \mathrm{cm}^{2}\right)$, following the Poisson distribution to account for illumination shot noise. We have assumed that the detection of electrons is perfect and does not introduce additional noise.

We measure line edge roughness (LER) in simulated top-down SEM images of randomly generated rough lines by using PSD analysis. The measurements are then compared to the actual SWR that was used to generate the rough lines. We conclude that the bias in the determination of SWR is a non-linear function of the correlation length $\xi_{3 D}$ of the actual SWR. The measured correlation length $\xi_{2 D}$ shows a linear trend with the correlation length $\xi_{3 D}$ of the SWR. From another simulation run, we conclude that the relation between measured LER in the top-down image and the standard deviation $\sigma_{3 D}$ of the SWR is linearly biased. We see that the amount of bias relates to the correlation length $\xi_{3 D}$ of the SWR: The bias in the determination of SWR from top-down images increases for decreasing correlation length $\xi_{3 D}$ of the actual SWR. The results of this study, with respect to the metrology of rough resist features, touches upon the reliability and comparability of roughness characterization in top-down images.
\end{abstract}

*Electronic address: T.Verduin@tudelft.nl

Metrology, Inspection, and Process Control for Microlithography XXIX, edited by Jason P. Cain, Martha I. Sanchez, Proc. of SPIE Vol. 9424, 942405 - (c) 2015 SPIE

CCC code: $0277-786 X / 15 / \$ 18 \cdot$ doi: $10.1117 / 12.2085768$

Proc. of SPIE Vol. $9424942405-1$ 
Keywords: Metrology, Scanning electron microscopy, Line edge roughness, Sidewall roughness, Power spectral density, Monte Carlo methods

\section{INTRODUCTION}

In a previous numerical study, we proposed a method for the determination of line edge roughness (LER) in low dose top-down secondary electron microscopy images. ${ }^{1}$ However, the true size, shape and roughness characteristics of resist features are not fully investigated in the analysis of top-down SEM images. The reason is that in reality, rough resist features are complex threedimensional structures. The characterization of roughness of resist features naturally extends to the analysis of sidewall roughness (SWR) which can be measured, for instance, by using an atomic force microscope (AFM). However, in view of the large volume of wafers being produced in a typical production line, AFM is not considered as a suitable metrology tool. Another problem with AFM involves the complexity of the measurement, in which the exact shape of the tip plays a crucial role.

Numerical studies on SWR are difficult to perform because the details of the roughness are defined at the (sub) nanometer scale. Studies involving the discrete modeling of the roughness on the sidewalls are therefore subject to time consuming simulations, and especially in the case of MonteCarlo simulations. The studies that we found in literature indicate that the true SWR is larger than the measured LER in a top-down SEM image. ${ }^{2,3}$ We have a few remarks with respect to these studies. In the study of $\mathrm{Li}$ et al., ${ }^{2}$ the focus is on pure polycrystalline silicon lines with a Gaussian roughness model for the sidewalls. The roughness of a resist feature, however, is typically characterized by more than just the standard deviation of a Gaussian-like distribution and involves additional parameters, such as correlation length and a roughness exponent. ${ }^{1,4-6}$ The study of Lawson et al. ${ }^{3}$ is different for two reasons. In the first place, Lawson et al. have used a more sophisticated roughness model. ${ }^{7}$ Unfortunately, the details of the roughness parameters are not explicitly mentioned nor are they varied to study its influence on the measured LER. Second, the lines used in the study of Lawson et al. are made of pure polymethyl methacrylate (PMMA) coated on a pure silicon substrate. Not only is PMMA a different material, it also comes, contrary to pure silicon lines, with a risk of resist shrinkage caused by the electron beam. ${ }^{5}$ Although it is not addressed in the work of Lawson et al., this risk can be reduced, for example, by lowering the beam voltage and total electron dose, i.e. reduce the number of integration frames. ${ }^{1,5}$

We would like to extend the study of Refs. 2 and 3 and examine the relation between SWR and top-down measured LER by varying the parameters of a non-Gaussian roughness model. What happens, for example, to the measured LER when the correlation length of the SWR changes? In particular, we consider a case similar to that of Lawson et al., in which we have rough PMMA lines on silicon. However, in order to reduce the risk of shrinkage, we will consider a beam energy of $300 \mathrm{eV}$ and an approximate dose of 20 incident electrons per pixel. This is about the lowest possible setting with a standard CD-SEM. We have already shown in a previous study, albeit theoretically, that LER can be determined under such noisy circumstances. ${ }^{1}$ For this study, we use the power spectral density (PSD) function to model the roughness as described by Palasantzas, ${ }^{4}$

$$
\operatorname{PSD}(k)=\frac{\sqrt{\pi}}{2 \pi} \frac{\Gamma\left(\alpha+\frac{1}{2}\right)}{\Gamma(\alpha)} \frac{2 \sigma^{2} \xi}{\left(1+k^{2} \xi^{2}\right)^{\alpha+\frac{1}{2}}}
$$

which we have also used in our previous study and seems a logical extension to the case of rough surfaces. ${ }^{1}$ In this model, given by Eq. 1, roughness is defined with $\sigma$ as the standard deviation, $\xi$ the correlation length and $\alpha$ the roughness exponent. Random rough surfaces with a PSD equal to that of Eq. 1 are generated by using the method of Thorsos, which is explained for example in Ref. 6. The idea is to compute the inverse two-dimensional Fourier transform of the amplitude 
of the PSD with a random phase. We remark that the roughness generated via the method of Thorsos is biased. This can be corrected for by multiplying the resulting displacements of the roughness with a scalar, which is also explained in Ref. 6 . We emphasize that the statistics of the rough surfaces generated in this way are isotropic.

Although we have decided on a particular roughness model, we are still in need of a program to simulate the top-down images. We already foresee that computation time is an important issue, since we intend to vary the parameters of a roughness model and preferably generate some statistics as well. Fortunately, there are a handful of simulation programs found in literature. We distinguish between simulators that use stopping power relations and dielectric function theory for modeling inelastic scattering events. Examples of simulators based on stopping powers are JMONSEL ${ }^{8}$ (developed at NIST), and CASINO $^{9}$ (developed at Université de Sherbrooke). Then there are programs based on dielectric function theory. We mention the programs of Kieft et al. ${ }^{10}$ (developed at FEI company), Li et al. ${ }^{2}$ and Lawson et al. ${ }^{3}$ Unfortunately, most of these programs are not available to us and the programs that we do have, are not designed to manage the complex threedimensional geometrical features of this study. The program of Kieft et al. has drawn our special attention for two important reasons. First of all, it has refinements which are specifically intended for low-voltage SEM simulations, and second, the secondary electron yields are well validated against experiment. However, the simulator of Kieft and Bosch is facing the same problem as all other simulators in this field of research: Monte-Carlo simulations of electron-specimen interactions are time consuming. It is the latter that has led us to develop a home-built Monte-Carlo simulator by combining the computational power of multi-core CPUs and GPUs.

\section{SEM SIMULATOR}

The program that we have created for this study is a full Monte-Carlo electron-specimen interaction simulator. It is designed from scratch for the purpose of solving problems involving large number of incident low energy particles and complex geometries, while using the refinements as described in Ref. 10. We consider three physical processes for the electron in our simulator. There are (1) elastic scattering events, (2) inelastic scattering events and (3) boundary crossings from one material to another. For the elastic and inelastic scattering events we make use of cumulative differential inverse mean free paths (mfps) tabulated as a function of kinetic energy. We assume that the azimuthal scattering angle, for both elastic and inelastic events, is isotropic. The cumulative tables are interpolated for intermediate values as follows,

$$
\ln \lambda^{-1}(E)=\frac{\ln \lambda^{-1}\left(E_{1}\right) \ln \frac{E_{2}}{E}+\ln \lambda^{-1}\left(E_{2}\right) \ln \frac{E}{E_{1}}}{\ln \frac{E_{2}}{E_{1}}}
$$

where $E_{1} \leq E \leq E_{2}$. The differential of the elastic cross-section is taken with respect to the polar scattering angle,

$$
\lambda_{\text {elastic }}^{-1}(E, \theta)=2 \pi \rho_{A} \int_{0}^{\theta} \frac{\mathrm{d} \sigma_{\text {elastic }}}{\mathrm{d} \theta^{\prime}} \sin \theta^{\prime} \mathrm{d} \theta^{\prime}
$$

where $E$ is the kinetic energy, $\rho_{A}$ is the atomic density of the material. A random scattering angle is obtained by inverse sampling of the (normalized) cumulative angular distribution function,

$$
U=\frac{\lambda_{\text {elastic }}^{-1}(E, \theta)}{\lambda_{\text {elastic }}^{-1}(E)}
$$

where $0 \leq U \leq 1$ is a uniformly distributed random number. For the inelastic scattering events, we have used the dielectric function model,${ }^{11}$ which we briefly mention here. The total inelastic 
inverse mfp is calculated in that model as follows,

$$
\lambda^{-1}(E)=\frac{1}{\pi E} \int \mathrm{d} \omega \int_{q_{-}}^{q_{+}} \frac{\mathrm{d} q}{q} \operatorname{Im}\left[-\frac{1}{\epsilon(q, \omega)}\right]
$$

where $\omega$ is the total energy loss for the electron, $q$ the momentum transfer to a secondary electron and $\epsilon(q, \omega)$ is the expanded dielectric function. The latter can be expressed in a plasmon-pole approximation as follows,

$$
\operatorname{Im}\left[-\frac{1}{\epsilon(q, \omega)}\right]=\frac{\omega^{\prime}}{\omega} \operatorname{Im}\left[-\frac{1}{\epsilon(0, \omega)}\right]
$$

where $\epsilon(0, \omega)$ relates to optical data, which is readily available for a broad range of materials, and

$$
\omega^{\prime}=\omega-\frac{q^{2}}{2}
$$

is the dispersion relation with $\omega^{\prime}$ denoted as the zero-momentum energy transfer. In our implementation, we follow Ref. 10 and tabulate the cumulative differential inverse inelastic mfps, where the differential is taken with respect to zero-momentum energy transfer,

$$
\lambda_{\text {inelastic }}^{-1}\left(E, \omega^{\prime}\right)=\int_{0}^{\omega^{\prime}} \frac{\partial \lambda^{-1}(E)}{\partial \omega^{\prime \prime}} \mathrm{d} \omega^{\prime \prime}
$$

Similar to Eq. 4, we draw a random zero-momentum energy transfer by inverse sampling of the cumulative distribution function,

$$
U=\frac{\lambda_{\text {inelastic }}^{-1}\left(E, \omega^{\prime}\right)}{\lambda_{\text {inelastic }}^{-1}(E)}
$$

where $0 \leq U \leq 1$ is a uniformly distributed random number. A scattering event occurs when an electron has traveled a distance of

$$
s=-\lambda_{\text {total }} \ln U
$$

where $0<U<1$ is a uniformly distributed random number, and

$$
\lambda_{\text {total }}^{-1}=\lambda_{\text {elastic }}^{-1}+\lambda_{\text {inelastic }}^{-1}
$$

is the total inverse mfp. A scattering event is determined to be elastic whenever

$$
U<\frac{\lambda_{\text {elastic }}^{-1}}{\lambda_{\text {total }}^{-1}}
$$

where $0 \leq U \leq 1$ is a uniformly distributed random number.

With respect to the sources of our tabulated data for metals, semiconductors and insulators, we have strictly followed Ref. 10. This means, for example, that we have used the angular differential Mott cross-sections provided by Ref. 12 and the cross-sections based on low energy acoustic phonons ${ }^{13,14}$ for the elastic scattering events. The optical data for the inelastic scatter events are directly taken from the program of Ref. 10, where we also applied the refinements given in that article with respect to the inelastic scattering. In order to determine the binding energy of a secondary electron, we use the ionization cross-sections provided by Ref. 15 and the additional binding energies provided by the program of Ref. 10. The boundary crossing of electrons from one material to another involves a quantum mechanical transmission and reflection calculation as described by Ref. 16. If cross-sections for compound materials are not available, then we use a strategy similar to that of Ref. 10, i.e. the differential inverse mfps of the individual elements are summed. 
Our program accepts arbitrary three-dimensional geometries, which must be modeled in a special way. First of all, when an arbitrary geometry is given, it must be decomposed into tetrahedra, which is the fundamental element of volume assigned to a particular material of interest in our simulator. Second, the faces of adjacent tetrahedra must be connected via shared vertices. This means that, for any tetrahedron, the neighbors (four at maximum) are accessible via shared faces. The latter speeds up the computation, as electrons can easily scatter into neighboring elements, which are directly accessible from the element the electron is currently in. There is, however, a significant problem that blows up the computation time. The primary electrons, i.e. the electrons which are incident on the sample, are created in vacuo above the sample and are therefore not enclosed by the volume elements. Therefore, we must determine the intersection with the face, that represents the vacuum-material interface, of the first encountered element located in the direction of the electron. This is a costly procedure, because we must scan the entire hull of our geometry, which is the set of all faces with vacuum on one of the sides, and search for the first encountered intersection. Note that the number of faces that constitute the hull is of the same order as the number of volume elements, which we assume to be very large. Moreover, it is possible that electrons scatter in and out of various volume elements with vacuum in between. This means that the intersection with the hull is determined at least once for all particles, but multiple times for the electrons that scatter from one material to vacuum and into another material again. We conclude that we prefer to avoid this procedure.

We propose another solution in which we decompose not only the geometry into tetrahedra, but also the vacuum. This means that the electrons may scatter freely in and out of vacuum, which is a volume element in this case, while avoiding the costly determination of hull intersections. Although it seems that we have solved the hull problem, we have exchanged this problem for another. For every primary (incident) electron, we must scan through all elements assigned to vacuum in order to find the element at which the electron is located. Fortunately, this is not really a problem for two reasons. First of all, the calculation must be performed exactly once at the beginning of the simulation and, second, the calculation can be offloaded to a GPU, which is dedicated to solving problems like this. The computational cost of this procedure renders therefore negligible in comparison to the total computation time required for simulating an entire image.

Although our program is very similar to that of Ref. 10, there are three differences to be mentioned. First of all, our program does not use the Geant4 platform. Instead we have developed and implemented our own manager for tracking and transporting electrons through matter. Second, we did not, for the purpose of this study, implement the Auger-effect. By calculating yield plots with the program of Ref. 10, we found that the significance of the Auger-effect on low energy secondary electron yields for silicon and PMMA is less than $1 \%$. Finally, there is no cutoff energy for electrons and we keep tracking all electrons until they run out of energy, even when they have little or no chance of escaping the material.

In order to verify that our simulator produces results similar to that of Kieft et al., we compare the secondary electron yields. In Fig. 1 we see that the agreement with silicon is satisfactory, for both the value of the yield and the energy at which the maximum yield occurs. The energy at which the maximum yield of PMMA occurs is also satisfactory. The value, however, of the yield for PMMA at the maximum is off by approximately $15 \%$. At the moment, we have no clear explanation for this particular anomaly. We do not, however, consider this a real problem, because we are interested in simulating electron energies $\leq 300 \mathrm{eV}$ and we see that the yield plot for PMMA in this lower energy range is satisfactory in agreement. 


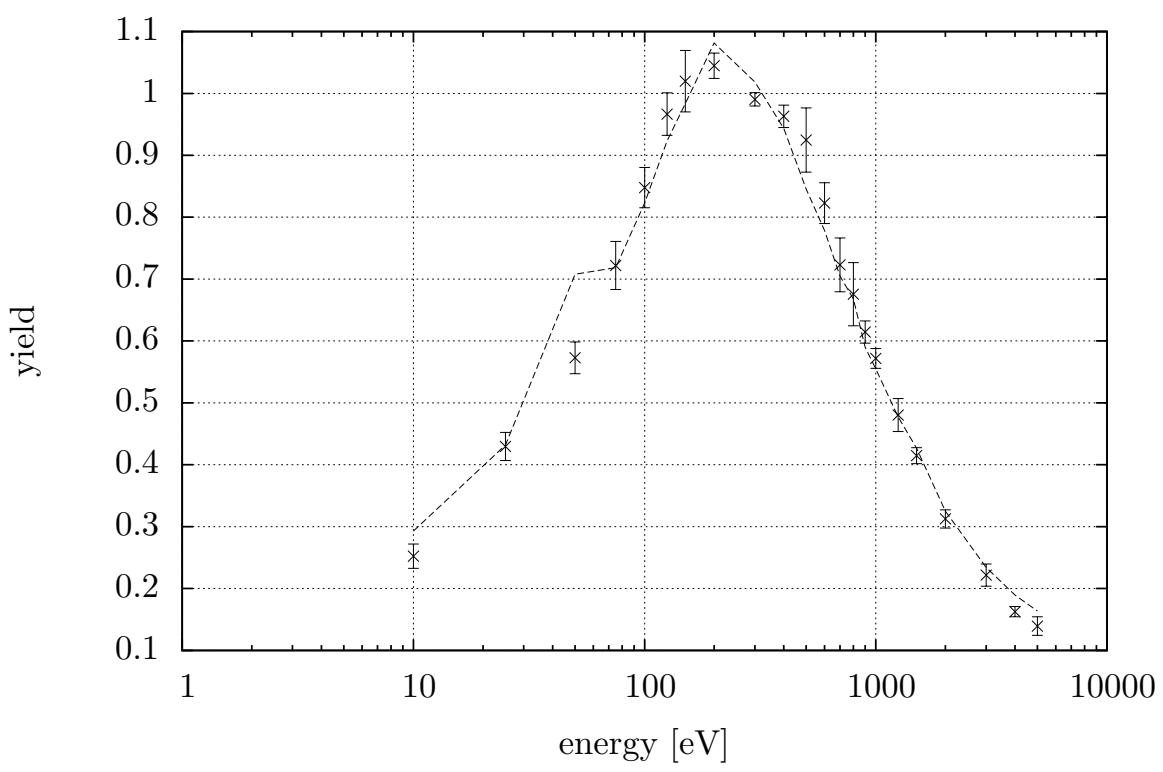

(a) Simulated secondary electron yield for silicon.

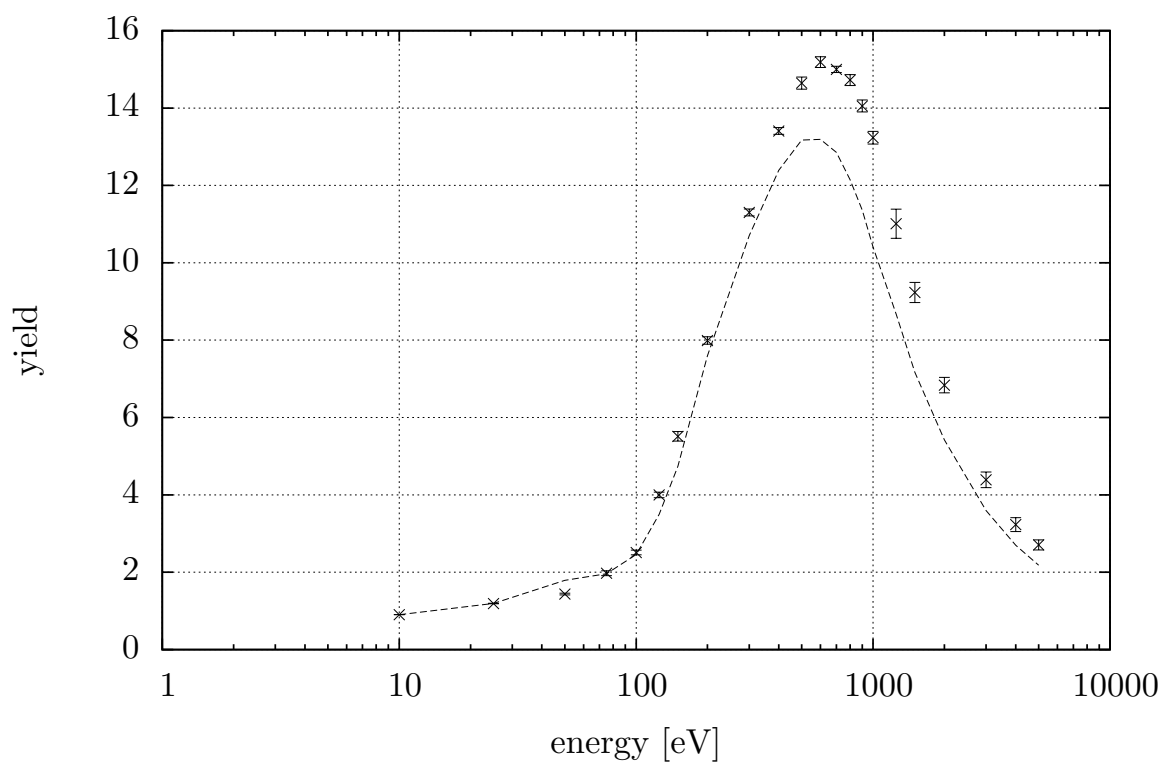

(b) Simulated secondary electron yield for PMMA.

Figure 1: Simulated secondary electron yields for silicon (a) and PMMA (b). The cross markers with error bars represent the result calculated using our home-built program. The dashed line is the result from the program of Kieft et al. Each marker corresponds to a total of 5 simulations in which a 1000 primary electrons are incident on the infinite flat surface of an infinite thick material. The total number of secondary electrons emitted from the surface with an energy less than $50 \mathrm{eV}$ are counted. The given yield is equal to the counted number of secondary electrons divided by the total number of incident primary electrons. 


\section{SIMULATION OF ROUGH LINES}

We will study a pattern of rough lines and spaces, where each line is made of PMMA located on a silicon substrate. The dimensions of the lines are $2 \mu \mathrm{m}$ in length, $32 \mathrm{~nm}$ wide and $32 \mathrm{~nm}$ in height. We randomly generate rough lines as follows. At first we create a template line with flat surfaces of which, for example, a small section is shown in Fig. 2a. A randomly generated rough surface is made by using the PSD of Palasantzas, given by Eq. 1, and the method of Thorsos. The area of the rough surface is equal to that of the flat sidewall $(2 \mu \mathrm{m} \times 32 \mathrm{~nm})$. We define the rough surface with a resolution of $1 \mathrm{~nm}$ in both dimensions. The justification for this number will be given later, when we define the image pixel size and beam spot size. An example of a randomly generated rough surface is shown in Fig. 2b. Rough lines are produced by replacing the flat sidewalls of the line shown in Fig. 2a with randomly generated rough surfaces. The result of that is illustrated in Fig. 2c. We emphasize that the top of the PMMA line remains flat, and the silicon substrate remains flat too. This is analogous to the work of Li et al., and Lawson et al. In reality, the top of the line and the substrate are expected to be rough as well. In that view, for more realistic cases, we should include the effects of post litho processing as well. The effect of that could be, for instance, that the base of the sidewall is inclined or even curved. None of these effects are taken into account in this study, which means that we investigate a rather ideal case of SWR.

The randomly generated rough line is decomposed into tetrahedra, such that it can be used in our home-built simulator. That sounds easy, but let us pause here for a moment, because the decomposition of a rough line into an acceptable system of tetrahedra for our simulator is not a trivial exercise. It is non trivial due to the special requirement for neighboring elements to have shared faces and thus shared vertices. In reality, we decompose the rough line into hexahedra at first, and then the hexahedra are decomposed into tetrahedra. The hexahedra must be alternating as the decomposition of a hexahedron into tetrahedra has two possible variants. The alternating series emerges because both variants are required in order to match the faces and vertices of the resulting system of tetrahedra in a proper way. To complicate matters even further, we do not use a single isolated line, but instead, we merge three randomly generated lines next to each other with a spacing of $32 \mathrm{~nm}$ in between. The reason for this more complicated geometry (which has approximately three times the number of elements), is to accommodate for the scattering of electrons into neighboring lines. Now that we have the sample defined, we can instruct the program to only scan the middle line and count the secondaries as follows. An electron is instantaneously detected whenever the following two criteria are satisfied: (1) The electron is transmitted from material to vacuum and (2) the kinetic energy of the electron is less than $50 \mathrm{eV}$. We have assembled illustrations of induced scattering events as calculated by our home-built simulator in Fig. 3.

Let us now discuss the actual simulation of an image of a randomly generated rough line. The pixel size is fixed at the size of $0.43 \mathrm{~nm} \times 2.7 \mathrm{~nm}$ (width times height), which is in accordance with our previous study of rough lines. ${ }^{1}$ We choose a beam with an energy of $300 \mathrm{eV}$ and a spot size of $3 \mathrm{~nm}$. The decision for this spot size (instead of a smaller one) is due to aberrations related to the low energy of the beam. The decision for the low beam energy is two-fold, (1) to reduce the risk of shrinkage that is involved in samples made of organic resist, such as PMMA, and (2) to reduce the computation time. Note that the spot size and the pixel size in the direction of the edges are larger than the resolution of $1 \mathrm{~nm}$ at which the rough surfaces of the sidewalls are defined. Each pixel is exposed with 20 electrons on average following the Poisson distribution, by which we simulate the effect of illumination shot noise. The resulting dose is approximately $276 \mu \mathrm{C} / \mathrm{cm}^{2}$ on average. Our sample is made of three randomly generated rough lines next to each other of which we only image an area of $64 \mathrm{~nm} \times 1 \mu \mathrm{m}$ (width times length) of the middle one. An example of a simulated SEM image of a randomly generated rough line is shown in Fig. 4. There are a few remarks to be made with respect to this simulated image. In the first place, we have not simulated the effect of detector noise, i.e. the detection of electrons is assumed to be perfect. For a more realistic image we should 


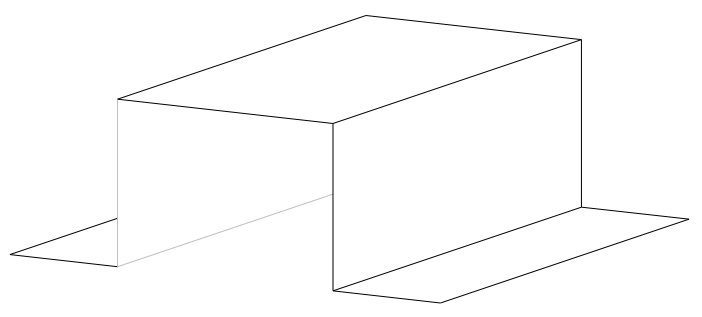

(a) A small section of a line with flat surfaces is shown. This flat line is used as a template for the construction of a rough line.

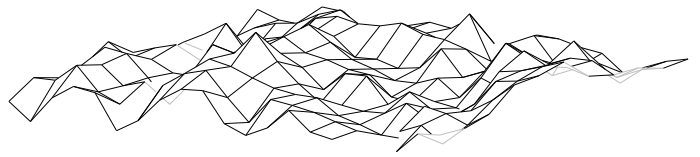

(b) A small section of a randomly generated rough surface is shown. The roughness is generated by effectively sampling displacements over a discretized flat surface by using the PSD function of Palasantzas and the method of Thorsos. This surface has a roughness defined with a standard deviation $\sigma_{3 D}$ of $1 \mathrm{~nm}$, correlation length $\xi_{3 D}$ of $20 \mathrm{~nm}$ and a roughness exponent $\alpha_{3 D}$ of 0.75 . The width and height of the rough surface is equal to the full size of the sidewall of the flat line of which a small section is illustrated in (a).

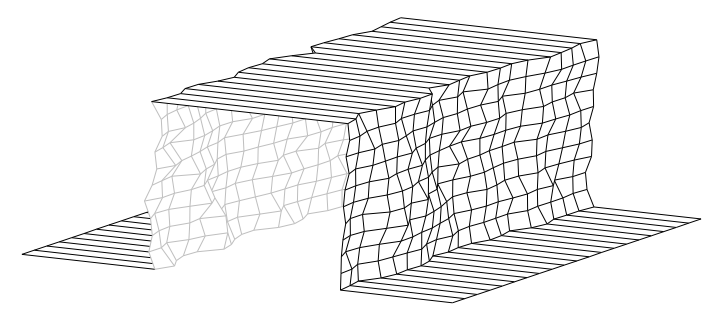

(c) A small section of a randomly generated rough line is shown. The flat sidewalls shown in (a) are replaced with rough surfaces, of which one example is shown in (b).

Figure 2: The construction of a randomly generated rough line is demonstrated. At first, a flat line (a) with a length of $2 \mu \mathrm{m}$, width of $32 \mathrm{~nm}$ and a height of $32 \mathrm{~nm}$ is constructed. The sidewalls of the flat line are replaced with randomly generated rough surfaces (b). The result of that is a randomly generated rough line of which a small section is shown in (c). Note that the top of the line and the substrate, on which the line is located, remains flat. 


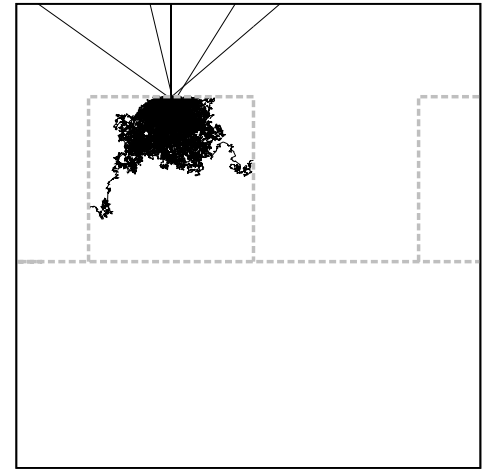

(a) Illustration of the scattering events induced by 20 primary electrons with an energy of $300 \mathrm{eV}$ which are incident at the center of a flat line (PMMA) on a silicon substrate. The line and substrate are shown by the dashed line.

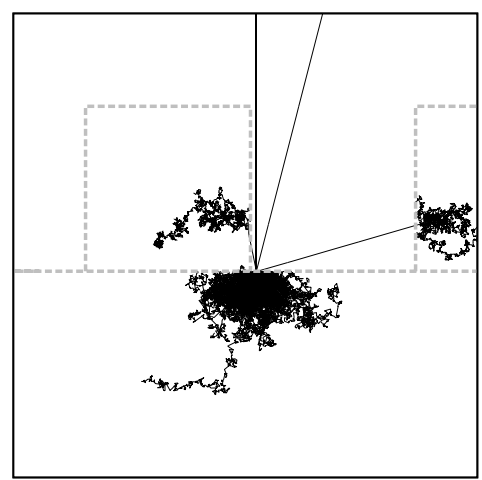

(c) Illustration of the scattering events induced by 20 primary electrons with an energy of $300 \mathrm{eV}$ which are incident just after the edge of a line (PMMA) into the silicon substrate. The line and substrate are shown by a dashed line. Notice that backscattered electrons from the substrate are traveling through vacuum into the neighboring lines where more scattering events are induced.

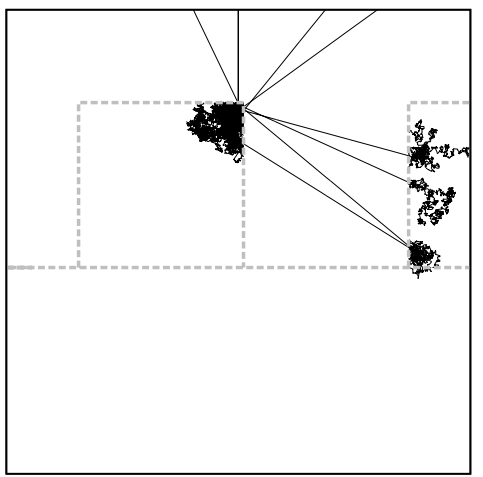

(b) Illustration of the scattering events induced by 20 primary electrons with an energy of $300 \mathrm{eV}$ which are incident just before the edge of a flat line (PMMA) on a silicon substrate. The line and substrate are shown by a dashed line. Notice that backscattered electrons from the incident line are traveling from the right edge, through vacuum into a neighboring line where more scattering events are induced.

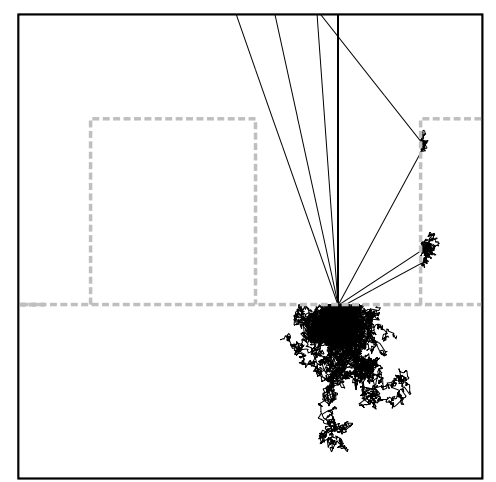

(d) Illustration of the scattering events induced by 20 primary electrons with an energy of $300 \mathrm{eV}$ which are incident into the silicon substrate between two lines (PMMA). The line and substrate are shown by a dashed line. Notice that backscattered electrons from the substrate are traveling through vacuum into the line at the right where more scattering events are induced.

Figure 3: Demonstration of the scattering events induced by an incident stream of primary electron into a sample of lines and spaces at different positions. The detection of secondary electrons with an energy less than $50 \mathrm{eV}$ at the surface of the lines and substrate is not shown. In reality, the lines have rough sidewalls and the electron beam has a finite spot size of $3 \mathrm{~nm}$. 
include, for example, a detection threshold, detection efficiency, Gaussian-like background noise and additional Poisson noise. The precise position of a detector also plays a role in the image formation as the position of the detector could introduce a shadow effect. This means that we expect to find more noise in a real SEM image with 20 electrons per pixel on average than shown in Fig. 4. Moreover, in a real (CD-)SEM, there is also a control mechanism for image contrast. We, however, simply scaled the intensity linearly over the full range of secondary counts. Although none of these additional effects in the image formation are taken into account in our simulations, the resulting image, i.e. Fig. 4 still appears realistic.

In order to generate statistics, we repeat the simulation of a randomly generated rough line 10 times. This means that, for each roughness, we calculate a full image of rough lines and spaces such as shown in Fig. 5. The resulting image is used for analysis by applying the method as presented in our previous study. ${ }^{1}$ In that method we (1) measure the edge displacements using a model for the integrated profile function, (2) determine the PSD by Fourier transform and finally (3) fit the measured PSD against Eq. 1 extended with a white noise term in order to capture the pixel noise. Let us discuss the two examples of the PSD analysis shown in Fig. 6. The SWR that was generated for both cases has an equal standard deviation $\sigma_{3 D}$ of $1 \mathrm{~nm}$ and equal roughness exponent $\alpha_{3 D}$ of 0.75 . The correlation length, however, was taken differently. In Fig. 6a we have used a correlation length $\xi_{3 D}$ of $6 \mathrm{~nm}$, and for Fig. 6b we have used a correlation length $\xi_{3 D}$ of $25 \mathrm{~nm}$. First of all, the bending point at the far left shifts in the direction of lower frequencies as the correlation length increases. This means that, in the case of the increased correlation length, the high frequencies, relative to the low frequencies, are more suppressed. This can also be seen from Eq. 1, where the correlation length $\xi$ is coupled to the wavenumber $k$. Suppose, for the sake of argumentation, that the PSDs in Fig. 6 are unbiased measurements of the actual SWR. Note that the total variance $\sigma_{3 D}^{2}$ is obtained by integrating Eq. 1 . Since the standard deviation $\sigma_{3 D}$ is kept constant, the area under the PSDs must be equal as well. As we increase the correlation length, the PSD essentially shifts to the left, due to the coupling with wavenumber $k$, and the total area remains invariant because of an increase in power. This can also be seen from Eq. 1, where the power (and thus the integral, hence the variance) scales as a function of correlation length $\xi$.

Although the (pixel) noise is found to be close to equal $(0.5 \mathrm{~nm})$, the measured LER for Fig. 6a $(0.63 \mathrm{~nm})$ is smaller than for Fig. $6 \mathrm{~b}(0.90 \mathrm{~nm})$. This means that the determination of SWR from top-down SEM images is a biased estimation. Since the actual three-dimensional roughness has a standard deviation $\sigma_{3 D}$ of $1 \mathrm{~nm}$, we conclude that the bias in LER has increased for decreasing correlation length $\xi_{3 D}$. 

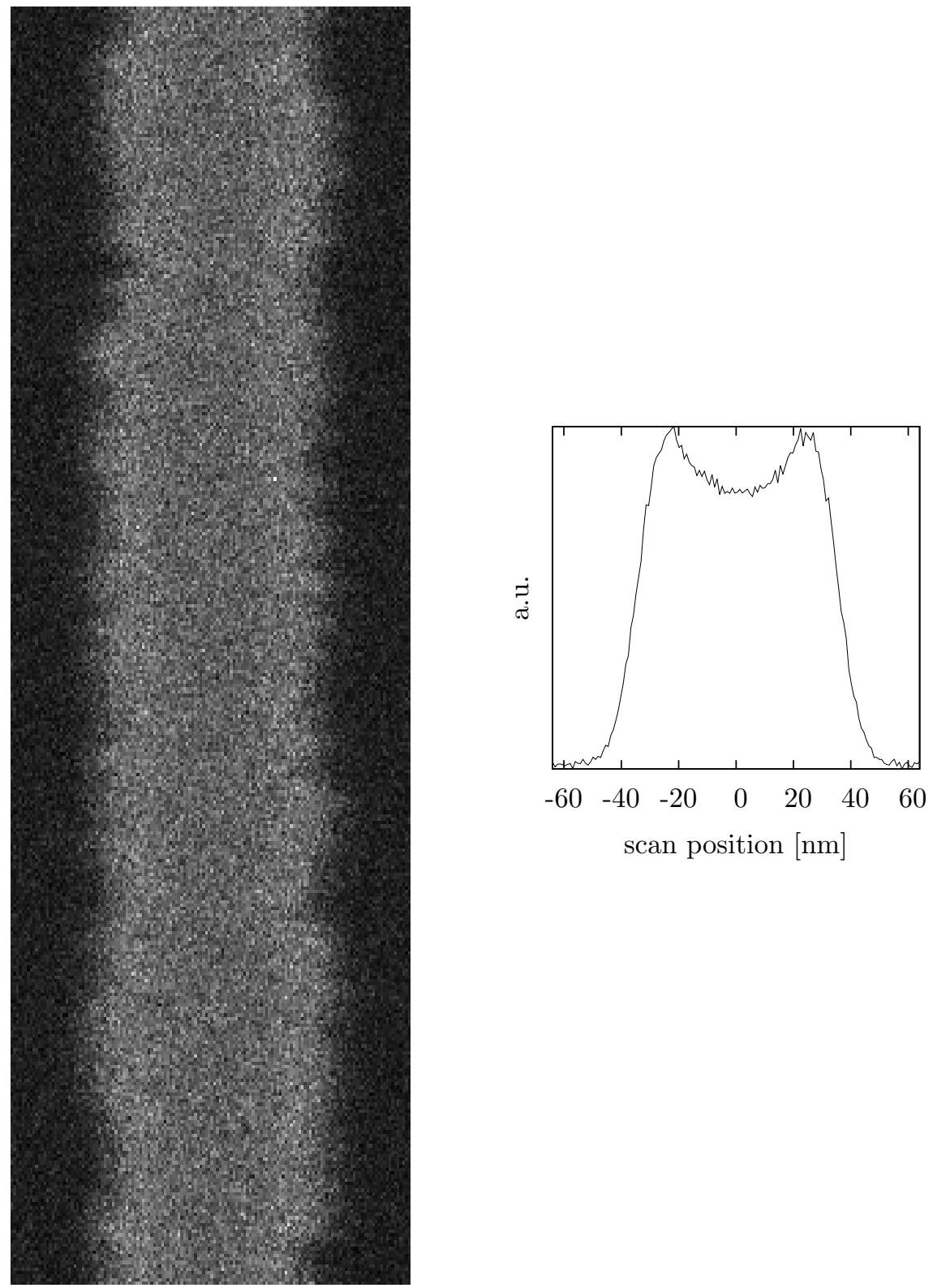

Figure 4: Simulated top-down image of a randomly generated rough line (left), including its integrated profile (right) is shown. The line is made of PMMA, located on a silicon substrate and is $1 \mu \mathrm{m}$ long, $32 \mathrm{~nm}$ wide and $32 \mathrm{~nm}$ in height. The area of the image is $64 \mathrm{~nm} \times 1 \mu \mathrm{m}$ (width times length). The SWR that was generated has a standard deviation $\sigma_{3 D}$ of $1.5 \mathrm{~nm}$, correlation length $\xi_{3 D}$ of $20 \mathrm{~nm}$ and a roughness exponent $\alpha_{3 D}$ of 0.75 . The image of the line is not from an isolated line, but is actually the middle one of three lines which are located next to each other with $32 \mathrm{~nm}$ spacing in between. The pixel size is $0.43 \mathrm{~nm} \times 2.7 \mathrm{~nm}$ (width times height). Each pixel is exposed with 20 primary electrons on average by sampling the Poisson distribution. The primary beam has a spot size of $3 \mathrm{~nm}$ and the kinetic energy of the primary incident electrons equals $300 \mathrm{eV}$. The detector for secondary electrons with an energy less than $50 \mathrm{eV}$ is assumed to be perfect and does therefore not introduce additional noise. 

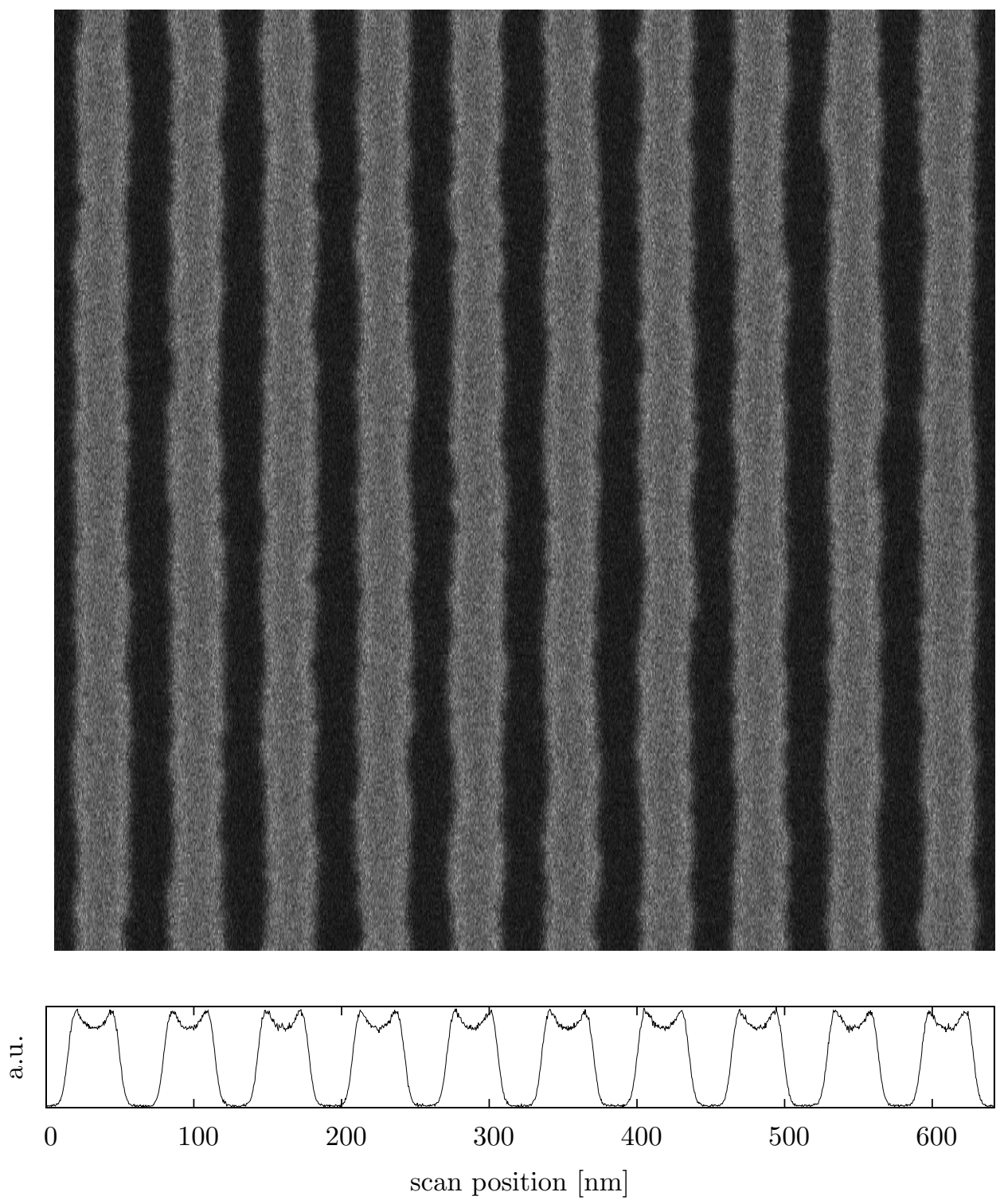

Figure 5: Simulated top-down image of randomly generated rough lines and spaces (top), including its integrated profile (bottom) is shown. This image is constructed by merging the individual images of ten randomly generated lines of which one is shown in Fig. 4. Each line is made of PMMA, located on a silicon substrate and is $1 \mu \mathrm{m}$ long, $32 \mathrm{~nm}$ wide and $32 \mathrm{~nm}$ in height. The SWR that was generated has a standard deviation $\sigma_{3 D}$ of $1.5 \mathrm{~nm}$, correlation length $\xi_{3 D}$ of $20 \mathrm{~nm}$ and a roughness exponent $\alpha_{3 D}$ of 0.75 . The pixel size of this image is $0.43 \mathrm{~nm} \times 2.7 \mathrm{~nm}$ (width times height). Each pixel is exposed with 20 primary electrons on average by sampling the Poisson distribution. The primary beam has a spot size of $3 \mathrm{~nm}$ and the kinetic energy is set to $300 \mathrm{eV}$. The detector for secondary electrons with an energy less than $50 \mathrm{eV}$ is assumed to be perfect and does therefore not introduce additional noise. 


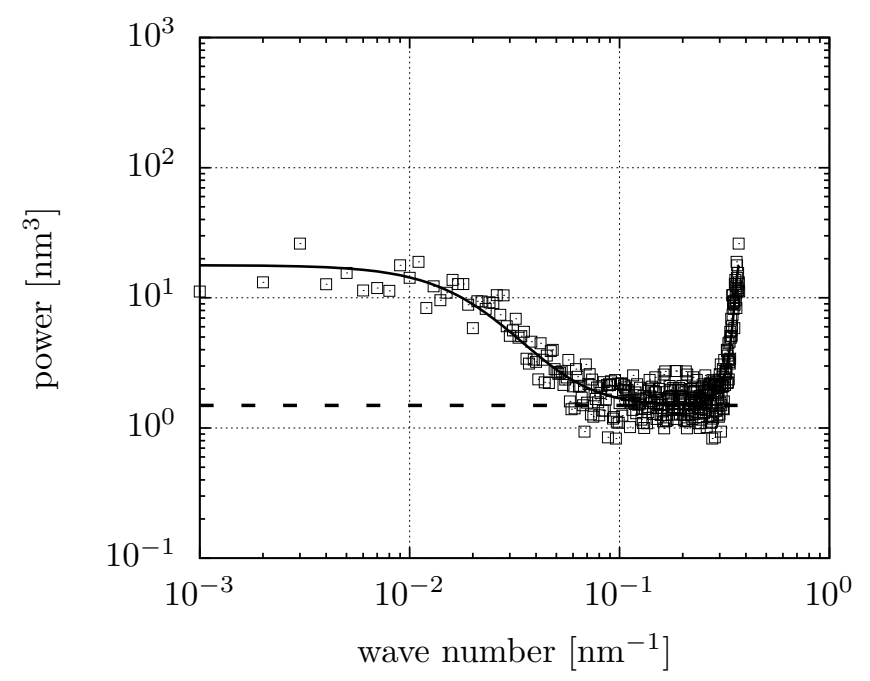

(a) Measured PSD, including a model fit, from a simulated top-down image of randomly generated rough lines and spaces is shown. The open squares are the measurements obtained from measuring ten lines, the solid line is the model fit (Palasantzas) and the dashed line is the corresponding (pixel) noise level. The SWR that was generated has a standard deviation $\sigma_{3 D}$ of $1 \mathrm{~nm}$, correlation length $\xi_{3 D}$ of $6 \mathrm{~nm}$ and the roughness exponent $\alpha_{3 D}$ was set to the value of 0.75 . The measured LER (one-sigma) found by fitting equals $0.63 \mathrm{~nm}$ with a pixel noise of $0.54 \mathrm{~nm}$

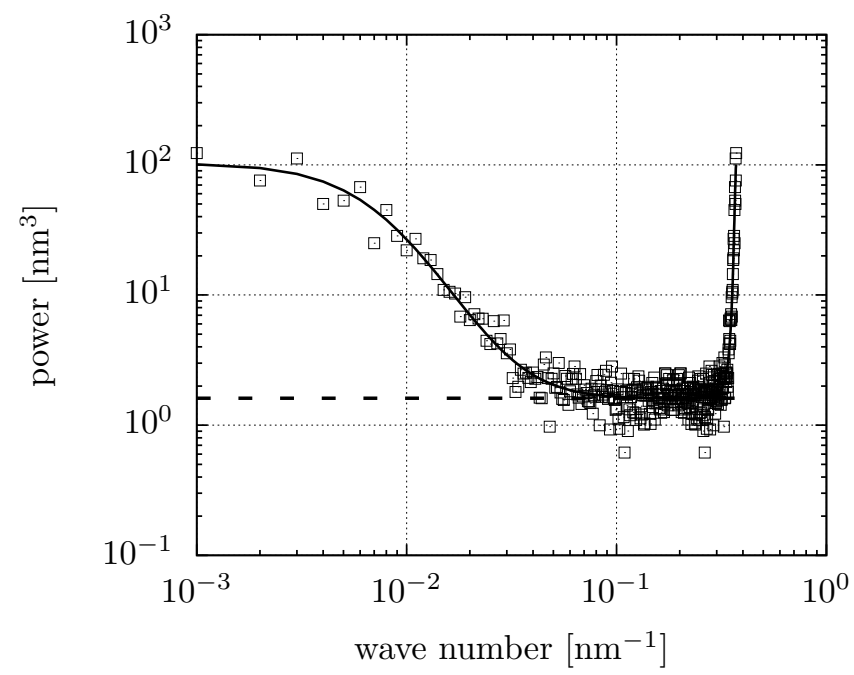

(b) Measured PSD, including a model fit, from a simulated top-down image of randomly generated rough lines and spaces is shown. The open squares are the measurements obtained from measuring ten lines, the solid line is the model fit (Palasantzas) and the dashed line is the corresponding (pixel) noise level. The SWR that was generated has a standard deviation $\sigma_{3 D}$ of $1 \mathrm{~nm}$, correlation length $\xi_{3 D}$ of $25 \mathrm{~nm}$ and the roughness exponent $\alpha_{3 D}$ was set to the value of 0.75 . The measured LER (one-sigma) found by fitting equals $0.90 \mathrm{~nm}$ with a pixel noise of $0.56 \mathrm{~nm}$.

Figure 6: Measurement and fitting of the PSD for a full image of randomly generated lines and spaces, see for example Fig. 4. 
Now that we are able to create, simulate, measure and analyze randomly generated rough lines, we demonstrate and discuss our simulations in which we vary the parameters of the Palasantzas roughness model as given by Eq. 1. In view of time, we have decided to restrict ourselves to simulate only the following two cases. At first we vary the correlation length $\xi_{3 D}$ of the SWR while keeping the standard deviation $\sigma_{3 D}$ at the fixed value of $1 \mathrm{~nm}$. The result of that simulation is shown in Fig. 7. We already concluded that the determination of SWR in top-down SEM images is biased when changing the correlation length of the SWR. Here, in Fig. 7, we see that the bias in SWR determination is actually a non-linear function of the correlation length $\xi_{3 D}$ of the SWR. The conclusion from the work of Lawson et al. was that LER is typically $50 \%$ smaller than the true SWR. In our simulation, this corresponds to a case where $\xi_{3 D}<5 \mathrm{~nm}$. In order to compare, we must know the effective correlation length $\xi_{3 D}$ for the SWR of PMMA in the mesoscopic roughness model of Lawson et al. Unfortunately, the parameters that they have used in the mesoscopic model for PMMA are not mentioned in Ref. 3 and a direct comparison can therefore not be made. In any case, the result of Fig. 7 indicates that the effective correlation length $\xi_{3 D}$ for the SWR of PMMA in the mesoscopic roughness model of Lawson et al. is probably less than $5 \mathrm{~nm}$. The two-dimensional measured correlation length $\xi_{2 D}$ in Fig. 7 indicates a linear relation with $\xi_{3 D}$. At the moment, we have no clear explanation for the sudden increase of the error bars for $\xi_{3 D} \geq 15 \mathrm{~nm}$. However, for the values $\xi_{3 D}<15 \mathrm{~nm}$ we see that the bias between measured $\xi_{2 D}$ and the correlation length $\xi_{3 D}$ of the actual SWR is on the order of a nanometer or two.

In a second simulation run, we fix the correlation length $\xi_{3 D}$ of the SWR to a value of $20 \mathrm{~nm}$ and vary the standard deviation $\sigma_{3 D}$. The result of that simulation is shown in Fig. 8. The solid line in Fig. 8 corresponds to the ideal case where LER $=\sigma_{3 D}$. We conclude that the relation between measured LER and the standard deviation $\sigma_{3 D}$ of the SWR is linearly biased. This was also the conclusion from the work of $\mathrm{Li}$ et al. The amount of bias, i.e. deviation from the solid line in Fig. 8, relates to the correlation length $\xi_{3 D}$ of the SWR, which is best seen in the results shown in Fig. 7. As we decrease the correlation length $\xi_{3 D}$ of the SWR, the bias in the determination of SWR increases non-linear.

For completeness, let us present the counts and timings of the simulations. The total number of simulated top-down images of rough lines and spaces, such as Fig. 5, equals 18. One image is made of approximately 19 million tetrahedra with 4.5 million shared vertices. Each image was simulated using approximately 11 million electrons on average. We have used three computers of which one has 6 cores and two have 4 cores, resulting in a total of 14 running cores. The total simulation time equals approximately 4 days.

So far we were unable to explain the biased results shown in Figs. 7 and 8. Moreover, now that we have seen that the bias in the determination of SWR from top-down SEM images can be very significant, we also question the dependence on other parameters. For example, is there any bias induced due to the electron beam energy? What happens to Fig. 7 as we increase the beam energy? What is the influence of the height of the lines and the penetration depth of the electrons in the material? These are important and fundamental questions with respect to the metrology as they touch upon the reliability and comparability of roughness characterization in top-down images. Although the results of this study are promising, we conclude that a follow up study is required for a deeper understanding. 

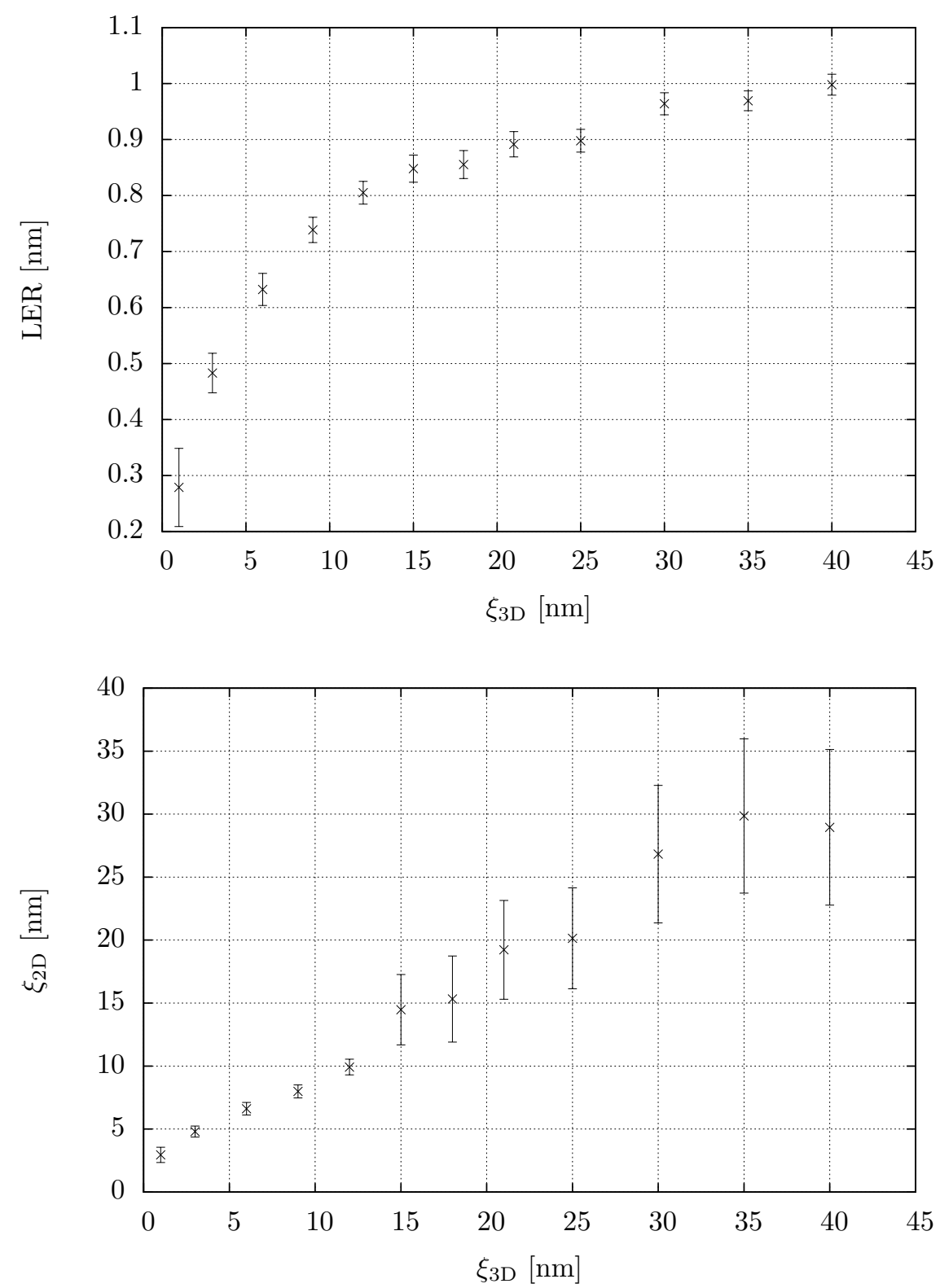

Figure 7: Measured LER (one-sigma) and measured correlation length $\xi_{2 D}$ versus the correlation length $\xi_{3 D}$ of the SWR. The markers are the result of analyzing the PSDs of randomly generated rough lines. The standard deviation $\sigma_{3 D}$ of the SWR is fixed at the value of $1 \mathrm{~nm}$, and the roughness exponent $\alpha_{3 D}$ is fixed at the value of 0.75 .

Proc. of SPIE Vol. $9424942405-15$ 


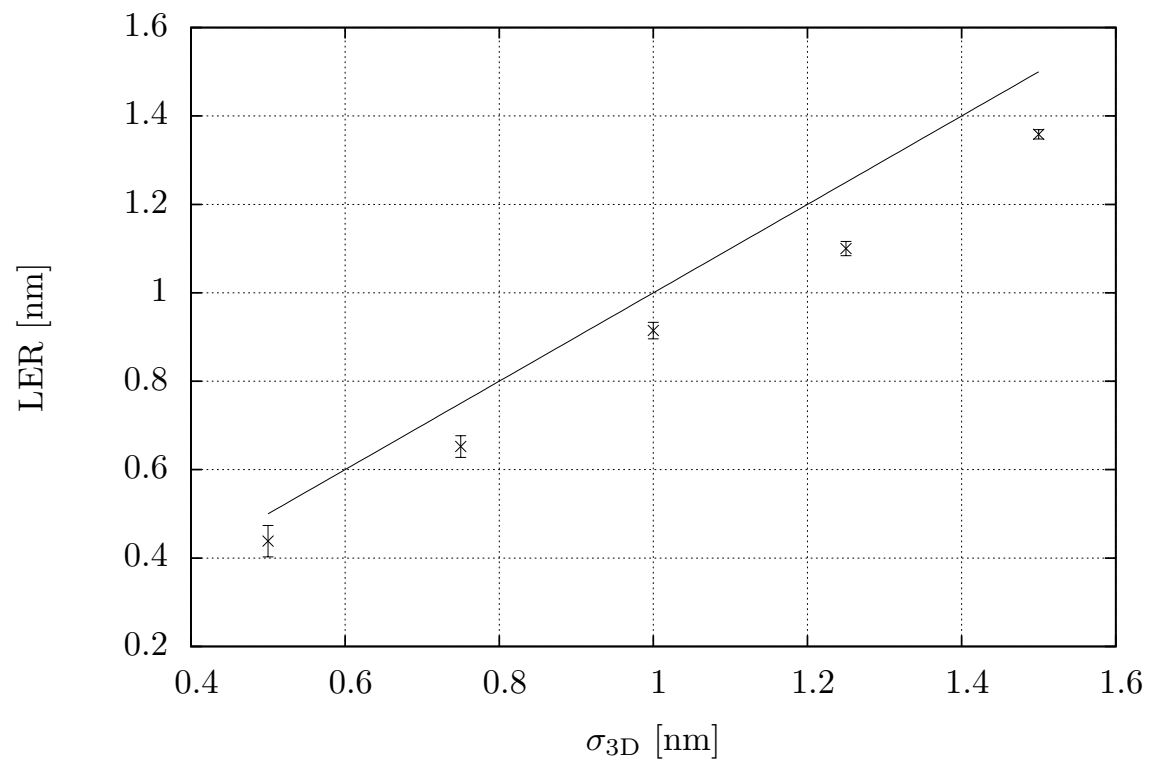

Figure 8: Measured LER (one-sigma) versus the standard deviation $\sigma_{3 D}$ of the SWR. The markers are the result of analyzing the PSDs of randomly generated rough lines. The solid line is the exact relation LER $=\sigma_{3 D}$ and demonstrates the bias in SWR determination. The correlation length $\xi_{3 D}$ of the SWR is fixed at the value of $20 \mathrm{~nm}$, and the roughness exponent $\alpha_{3 D}$ is fixed at the value of 0.75.

Proc. of SPIE Vol. $9424942405-16$ 


\section{CONCLUSION}

We have investigated the determination of SWR from top-down SEM images by using a numerical study. For the purpose of creating top-down SEM images, we have developed, from scratch, a home-built program for simulating electron-specimen interactions. The program that we have developed is optimized for complex arbitrary geometries and large number of incident low energy primary electrons by using multi-core CPUs and GPUs. It is based on the dielectric function model for inelastic scattering events and strictly follows the refinements for low-voltage SEM as given by Kieft et al. We have compared and verified that the secondary electron yields from our home-built simulator are in agreement with the work of Kieft et al.

In particular, we consider patterns of randomly generated rough PMMA lines located on a silicon substrate. Our lines have a length of $2 \mu \mathrm{m}$, a width of $32 \mathrm{~nm}$ and a height of $32 \mathrm{~nm}$. The SWR is modeled by using the PSD of Palasantzas and generated by using the method of Thorsos. The rough surface is defined at a resolution of $1 \mathrm{~nm}$ in both dimensions. The top surface of our PMMA lines and the silicon substrate are modeled as flat. We merge three randomly generated lines next to each other with a spacing of $32 \mathrm{~nm}$ in between to account for the scattering of electrons into neighboring lines.

We generate a SEM-like image of randomly generated rough lines by using a fixed pixel size of $0.43 \mathrm{~nm} \times 2.7 \mathrm{~nm}$ (width times height), which is in accordance with our previous study on rough lines. In order to reduce the risk of shrinkage, we use a beam energy of $300 \mathrm{eV}$ and a spot size of $3 \mathrm{~nm}$, which is due to the aberrations caused by the low energy of the beam. Each pixel is exposed with 20 electrons on average, following the Poisson distribution to account for illumination shot noise. The resulting dose is approximately $276 \mu \mathrm{C} / \mathrm{cm}^{2}$ on average. We have assumed that the detection of electrons is perfect and does not introduce additional noise to the SEM images.

We measure LER by using the PSD analysis as presented in our previous work while changing the standard deviation $\sigma_{3 D}$ of the SWR and correlation length $\xi_{3 D}$ of the SWR in a controlled way. The measured LER is then compared against the actual SWR that was used to generate the rough lines. We conclude that the bias in the determination of SWR is a non-linear function of the correlation length $\xi_{3 D}$ of the actual SWR. The measured correlation length $\xi_{2 D}$ shows a linear trend with the correlation length $\xi_{3 D}$ of the SWR. A direct comparison with the work of Lawson et al. cannot be made because the details of that mesoscopic model for PMMA are not mentioned. From another simulation run, we conclude that the relation between measured LER and the standard deviation $\sigma_{3 D}$ of the SWR is linearly biased. That conclusion is in agreement with the work of $\mathrm{Li}$ et al. We see that the amount of bias relates to the correlation length $\xi_{3 D}$ of the SWR. The bias in the determination of SWR increases for decreasing correlation length $\xi_{3 D}$ of the actual SWR. We were not able yet to explain the dependence of the bias in the determination of SWR. We suggest to find an explanation in a follow up study and also investigate other potential dependencies of the bias, such as beam energy, influence of the height of the lines and the penetration depth of the electrons in the material.

This work is supported by NanoNextNL, a micro and nanotechnology program of the Dutch Government and 130 partners. We would like to thank Kieft \& Bosch from FEI company for using their SEM simulator, which is an essential ingredient of this work. Special thanks to Erik Kieft for the discussions that have followed from the development of our home-built program.

\section{References}

[1] Verduin, T., Kruit, P., and Hagen, C. W., "Determination of line edge roughness in low dose top-down scanning electron microscopy images," Journal of Micro/Nanolithography, MEMS, and MOEMS 13, 033009 (2014). 
[2] Li, Y. G., Mao, S. F., Li, H. M., Xiao, S. M., and Ding, Z. J., "Monte Carlo simulation study of scanning electron microscopy images of rough surfaces," Journal of Applied Physics $\mathbf{1 0 4}$ (2008).

[3] Lawson, R. A. and Henderson, C. L., "Understanding the relationship between true and measured resist feature critical dimension and line edge roughness using a detailed scanning electron microscopy simulator," Journal of Vacuum Science 83 Technology B: Microelectronics and Nanometer Structures 28, C6H34 (2010).

[4] Palasantzas, G., "Roughness spectrum and surface width of self-affine fractal surfaces via the K-correlation model," Physical Review B 48, 472-478 (1993).

[5] Azarnouche, L., Pargon, E., Menguelti, K., Fouchier, M., Fuard, D., Gouraud, P., Verove, C., and Joubert, O., "Unbiased line width roughness measurements with critical dimension scanning electron microscopy and critical dimension atomic force microscopy," Journal of Applied Physics 111, 084318 (2012).

[6] Mack, C. A., "Generating random rough edges, surfaces, and volumes.," Applied Optics 52, 1472-1480 (2013).

[7] Lawson, R. A. and Henderson, C. L., "Three-dimensional mesoscale model for the simulation of LER in photoresists," Proceedings of SPIE 7639, 76392G-76392G-10 (2010).

[8] Villarrubia, J. S., Ritchie, N. W. M., and Lowney, J. R., "Monte Carlo modeling of secondary electron imaging in three dimensions," in Advanced Lithography, 6518, 65180K-65180K-14 (2007).

[9] Hovington, P., Drouin, D., and Gauvin, R., "CASINO: A new Monte Carlo code in C language for electron beam interaction - Part I: Description of the program," Scanning 19, 1-14 (1997).

[10] Kieft, E. and Bosch, E., "Refinement of Monte Carlo simulations of electron-specimen interaction in low-voltage SEM," Journal of Physics D: Applied Physics 41, 215310 (2008).

[11] Ashley, J., "Interaction of low-energy electrons with condensed matter: stopping powers and inelastic mean free paths from optical data," Journal of Electron Spectroscopy and Related Phenomena 46, 199-214 (1988).

[12] Czyzewski, Z., MacCallum, D. O., Romig, A., and Joy, D. C., "Calculations of Mott scattering cross section," Journal of Applied Physics 68, 3066 (1990).

[13] Fitting, H. J., Schreiber, E., Kuhr, J. C., and von Czarnowski, A., "Attenuation and escape depths of low-energy electron emission," Journal of Electron Spectroscopy and Related Phenomena 119, 35-47 (2001).

[14] Schreiber, E. and Fitting, H., "Monte Carlo simulation of secondary electron emission from the insulator SiO2," Journal of Electron Spectroscopy and Related Phenomena 124, 25-37 (2002).

[15] Cullen, D., Perkins, S., and Seltzer, S., "Tables and Graphs of Electron Interaction Cross 10 eV to $100 \mathrm{GeV}$ Derived from the LLNL Evaluated Electron Data Library (EEDL), Z = 1 - 100," Lawrence Livermore National Laboratory Evaluated Electron Data Library UCRL-50400 31 (1991).

[16] Shimizu, R. and Ze-Jun, D., "Monte Carlo modelling of electron-solid interactions," Reports on Progress in Physics 55, 487-531 (1999). 